\title{
Analytical Approaches for Porous Media Geothermal Power Calculations ${ }^{1}$
}

\author{
Daniel T. Birdsell ${ }^{\mathrm{a}, *}$, Benjamin M. Adams ${ }^{\mathrm{a}}$, Jonathan D. Ogland-Hand ${ }^{\mathrm{a}}$, Jeffrey M. Bielicki ${ }^{\mathrm{b}}$, \\ Mark. R Fleming ${ }^{\mathrm{c}}$, Martin O. Saar ${ }^{\mathrm{a}, \mathrm{d}}$ \\ ${ }^{\mathrm{a}}$ Geothermal Energy and Geofluids Group, Department of Earth Sciences, ETH Zurich, Zurich, Switzerland \\ ${ }^{\mathrm{b}}$ Department of Civil, Environmental, and Geodetic Engineering, John Glenn College of Public Affairs, The \\ Ohio State University, Columbus, OH, U.S.A. \\ 'Department of Mechanical Engineering, Milwaukee School of Engineering, Milwaukee, WI, U.S.A. \\ ${ }^{d}$ Department of Earth and Environmental Sciences, University of Minnesota, Minneapolis, MN, U.S.A. \\ "Corresponding author daniel.birdsell@ colorado.edu
}

\begin{abstract}
:
Geothermal electricity generation may play a role in reducing greenhouse gas emissions and addressing climate change in a cost-effective manner. Reservoir equations for pressure and temperature must be coupled to a power cycle model to calculate electricity generation from a geothermal power plant. This work focuses on sedimentary basin geothermal power production, which relies on flow through porous and permeable aquifers in sedimentary basins. Previous work has used numerical reservoir simulators, but we introduce analytical reservoir solutions for reservoir impedance, wellbore heat loss, and reservoir heat depletion in this work. The reservoir impedance and wellbore heat loss solutions are combined with a power cycle model to calculate electricity generation. The reservoir heat depletion solution provides insight into the reservoir lifetime because electricity generation decreases with reservoir temperature. We compare the analytical and numerical approaches and discuss their implications for geothermal electricity generation from sedimentary basins. Both approaches have merits, and the comparison herein can guide those who want to understand geothermal electricity production.
\end{abstract}

Keywords:

Sedimentary Geothermal Electricity; Porous Media Analytical Solution; Electric Power Generation; Reservoir Impedance; Geothermal Reservoir Heat Depletion; Wellbore Heat Loss

Nomenclature:

\begin{tabular}{|c|l|c|l|}
\hline$A_{c}$ & Cross-sectional area & $r_{0}$ & Well radius \\
\hline$A_{r e s}$ & Conduction area of reservoir & $r_{1}, r_{2}$ & $\begin{array}{l}\text { Distance from well to } \\
\text { equipotential line (Sec. 2.1.3) }\end{array}$ \\
\hline$D$ & Well diameter & $t$ & Time \\
\hline$L$ & Well spacing & $t_{d}$ & Dimensionless time \\
\hline$M$ & Source-sink strength & $x$ & $\begin{array}{l}\text { Cartesian horizontal spatial } \\
\text { coordinate }\end{array}$ \\
\hline$P$ & Fluid pressure & $x_{0}$ & $\begin{array}{l}\text { Location of well/porous media } \\
\text { interface }\end{array}$ \\
\hline$Q_{\text {Loss }}$ & $\begin{array}{l}\text { Heat exchange from wellbore } \\
\text { to surroundings }\end{array}$ & $z$ & Vertical spatial coordinate \\
\hline$T_{e}$ & $\begin{array}{l}\text { Background geothermal } \\
\text { temperature }\end{array}$ & $\Gamma$ & Non-dimensional temperature \\
\hline$T_{i n j}$ & $\begin{array}{l}\text { Injection temperature } \\
\end{array}$ & $\Delta P$ & $\begin{array}{l}\text { Pressure difference in the } \\
\text { reservoir between the injection } \\
\text { well and the midpoint between } \\
\text { the injection and production } \\
\text { well }\end{array}$ \\
\hline
\end{tabular}

${ }^{1}$ The short version of the paper was presented at Applied Energy Symposium: MIT A+B, August 13-14, Boston, MA with paper ID APEN-MIT-2020_215. This paper is a substantial extension of the short version of the conference paper. 


\begin{tabular}{|c|c|c|c|}
\hline$T_{r}$ & Reservoir temperature & $\Delta P_{\text {tot }}$ & $\begin{array}{l}\text { Pressure difference in the } \\
\text { reservoir between the injection } \\
\text { well and the production well }\end{array}$ \\
\hline$T_{w}$ & Well wall temperature & $\Delta P_{\text {Loss }}$ & Frictional losses in well \\
\hline$V$ & Darcy velocity & $\beta$ & Wellbore heat transfer factor \\
\hline$V_{\text {res }}$ & Volume of reservoir & $\epsilon$ & Heat depletion term \\
\hline$a$ & $\begin{array}{l}\text { Equivalent radius of the } \\
\text { reservoir }\end{array}$ & $\theta$ & Inclination in radial coordinates \\
\hline$a_{A}$ & Area-based equivalent radius & $\kappa$ & permeability \\
\hline$a_{V}$ & $\begin{array}{l}\text { Volume-based equivalent } \\
\text { radius }\end{array}$ & $\lambda$ & $\begin{array}{l}\text { Inverse of the product of the } \\
\text { contact resistance and the } \\
\text { thermal conductivity }\end{array}$ \\
\hline$b$ & Reservoir thickness & $\mu$ & Fluid viscosity \\
\hline$c_{e f f}$ & $\begin{array}{l}\text { Specific heat of rock/water } \\
\text { mixture }\end{array}$ & $\bar{\mu}$ & $\begin{array}{l}\text { Effective fluid viscosity in } \\
\text { reservoir }\end{array}$ \\
\hline$C_{\text {Fluid }}$ & Working fluid heat capacity & $\xi$ & $\begin{array}{l}\text { Parameter that accounts for heat } \\
\text { capacity inside and outside } \\
\text { reservoir. Typically } 3 \text {. }\end{array}$ \\
\hline$e$ & Euler's number & $\rho$ & Fluid density \\
\hline$h$ & Enthalpy & $\rho_{\text {eff }}$ & Density of rock/water mixture \\
\hline$k_{e f f}$ & $\begin{array}{l}\text { Effective thermal conductivity } \\
\text { of saturated fluid/rock mixture }\end{array}$ & $\bar{\rho}$ & $\begin{array}{l}\text { Effective fluid density in } \\
\text { reservoir }\end{array}$ \\
\hline$\dot{m}$ & Mass flow rate (general) & $\varphi$ & Stream potential \\
\hline$\dot{m}_{t o t}$ & $\begin{array}{l}\text { Total mass flow rate through } \\
\text { well }\end{array}$ & $\omega$ & $\begin{array}{l}\text { Thermal diffusivity of } \\
\text { fluid/rock mixture }\end{array}$ \\
\hline$r$ & $\begin{array}{l}\text { Radial horizontal spatial } \\
\text { coordinate }\end{array}$ & & \\
\hline
\end{tabular}

\section{Introduction}

Substantially reducing or eliminating $\mathrm{CO}_{2}$ emissions from the electricity sector while simultaneously electrifying substantial portions of the heat and transportation sectors are important components of leastcost pathways that limit the global mean surface temperature increase to $2^{\circ} \mathrm{C}$ or less [Krey et al. 2014; Kriegler et al. 2014; IPCC 2018]. Geothermal power plants may be valuable in this effort because they emit little to no $\mathrm{CO}_{2}$ and can provide dispatchable electricity [Sepulveda et al. 2018, Bistline and Blanford 2020]. While much prior work investigating geothermal power generation focuses on naturally faulted and fractured rock (i.e., hydrothermal resources) or hot dry rock that are artificially stimulated (i.e., Enhanced Geothermal System (EGS) resources), it is also possible to generate electricity with naturally porous and permeable sedimentary basin resources [Adams et al., 2015; Banks and Harris 2018; Tester et al., 2006; U.S. DOE., 2019]. Prior work in this area estimated electricity generation from sedimentary basin geothermal power plants by coupling numerical reservoir simulators with power cycle models [Adams et al., 2014, 2015, 2020; Ezekiel et al., 2020; Fleming et al., 2020; Garapati et al., 2020; Randolph and Saar 2011]. In contrast, little to no work has utilized analytical solutions for reservoir performance in conjunction with power cycle models to estimate power generation.

Analytical solutions have advantages and disadvantages when compared to numerical methods. They are typically easier to apply than numerical solutions. Moreover, they tend to have fewer degrees of freedom, which may lead to less human error. Furthermore, it is possible to understand underlying relationships between variables by simply examining an analytical solution's mathematical form. The Ideal Gas Law provides a simple example of seeing a relationship by examining an equation; it is clear that the pressure is proportional to temperature and inversely proportional to the volume. Analytical solutions are computationally inexpensive, which means they can be used to explore a large parameter space and in pre-screening studies. On the other hand, analytical solutions often rely on simplifying assumptions and approximations, making them less suitable for some applications. Sometimes, analytical solutions are unknown and are difficult to derive 
Numerical solutions, which have become popular due to increased computational power in recent decades, also have advantages and disadvantages compared to analytical solutions. Numerical approaches can often relax the assumptions and approximations that some analytical solutions utilize, making them suitable for applications where such simplifications are not warranted. For example, numerical solutions are often able to handle problems with complex geometry, heterogeneity, or coupled physics (e.g., thermo-hydro-mechanical-reservoir simulation). However, this complexity also has its drawbacks. There can be many degrees of freedom and user choices, such as the specification of parameters, decisions about meshing, and application of boundary and initial conditions. More degrees of freedom may lead to more human error. However, the potential for human error can likely be decreased if users learn about the governing equations, numerical methods, and assumptions embedded within numerical simulators. Furthermore, human error may also be reduced by built-in error checking, which will stop a numerical solution from running if incompatible parameters are specified. Finally, numerical error, which can come from roundoff error and truncation error, is always present in numerical solutions.

This paper: 1) introduces the application of analytical solutions for representing sedimentary basin geothermal reservoirs and 2) compares results from those analytical solutions against results from numerical models to elucidate and further demonstrate the pros and cons of the two methods for representing the subsurface. Our application is primarily focused on using techniques to represent sedimentary basin reservoirs in coupled models that estimate power generation, but we also investigate reservoir heat depletion because this may be important in investigations of geothermal power generation over decadal periods (i.e., the lifetime of a geothermal power plant). One novel aspect of our work is the combination of the analytical reservoir solutions with the power cycle model to calculate power generation, which has not been done in the context of sedimentary basin geothermal to our knowledge. Given the tradeoffs between the analytical and numerical methods and the broad applications for coupled models that can estimate electricity generation from sedimentary basin geothermal power plants, it is impossible to claim that one method is always more favorable, precise, or accurate compared to the other. However, our results offer insights that can guide people who are deciding how to represent sedimentary basin geothermal reservoirs when investigating geothermal power generation. In Sec. 2, we introduce analytical solutions for reservoir impedance, heat loss from a wellbore, and reservoir heat depletion. Since we compare to a numerical solution and calculate power generation, we also review the numerical reservoir simulator and power cycle model from Adams et al. [2015], which remain largely unchanged from that work. In Sec. 3, we compare the analytical and numerical solutions and their effect on the predicted power generation and reservoir lifetime. In Sec. 4, we provide conclusions.

\section{Methods}

Our methodology uses analytical solutions to calculate electric power generation and offer insights into a geothermal reservoir's useful lifetime. These analytical solutions are also compared to a numerical solution, similar to the one in Adams et al. [2015]. The electric power generation is related to the produced fluid's flow rate and temperature. Therefore, in Secs. 2.1 and 2.2, we provide analytical solutions for the reservoir impedance and wellbore heat loss. The reservoir lifetime is related to reservoir heat depletion because power generation decreases with reservoir temperature. Therefore, in Sec. 2.3 we provide an analytical solution for reservoir heat depletion. Since comparison to the numerical solution is a key aspect of this study, we recap the numerical solution that we have previously used [Adams et al., 2015], which remains mostly unchanged in this work. Sec. 2.5 details how electric power generation is calculated. These electric power calculations use the same methodology as Adams et al. [2015], except that the reservoir impedance comes from the analytical solutions (Sec. 2.1), and wellbore heat loss is included (Sec. 2.2).

The key simulation parameters are given in Table 1.

TABLE I. MODEL ASSUMPTIONS AND PARAMETERS

\begin{tabular}{|l|l|}
\hline \multicolumn{1}{|c|}{ Parameter } & \multicolumn{1}{c|}{ Value } \\
\hline Geologic Temperature Gradient & $35^{\circ} \mathrm{C} / \mathrm{km}$ \\
\hline Reservoir Pressure & Hydrostatic \\
\hline Well Diameter $(D)$ & $0.41 \mathrm{~m}$ \\
\hline Well Spacing $(L)$ & $707 \mathrm{~m}$ \\
\hline Surface Temperature & $15^{\circ} \mathrm{C}$ \\
\hline Well Pattern & $1 \mathrm{~km}^{2}$ Inverted 5-spot \\
\hline Primary Geothermal Fluid & Water \\
\hline
\end{tabular}




\begin{tabular}{|l|l|}
\hline \multicolumn{1}{|c|}{ Parameter } & \multicolumn{1}{c|}{ Value } \\
\hline Secondary ORC Fluid & R245fa \\
\hline Flow rate $(\dot{m})$ & $\begin{array}{l}100 \mathrm{~kg} / \mathrm{s} \text { (reservoir heat depletion calcs.) } \\
\text { Maximized power (power production calcs.) }\end{array}$ \\
\hline Permeability $(\kappa)$ & $5 \cdot 10^{-14} \mathrm{~m}^{2}$ \\
\hline Reservoir thickness $(b)$ & $300 \mathrm{~m}$ \\
\hline Thermal Conductivity of saturated media & $2.1 \mathrm{~W} / \mathrm{m}-{ }^{\circ} \mathrm{C}$ \\
\hline Density of saturated media $\left(\rho_{e f f}\right)$ & $2485 \mathrm{~kg} / \mathrm{m}^{3}$ \\
\hline Heat capacity of saturated media $\left(c_{e f f}\right)$ & $1320 \mathrm{~J} / \mathrm{kg}-{ }^{\circ} \mathrm{C}$ \\
\hline Thermal conductivity of saturated media $\left(k_{e f f}\right)$ & $2.1 \mathrm{~W} / \mathrm{m}-{ }^{\circ} \mathrm{C}$ \\
\hline Injection temperature $\left(T_{\text {inj }}\right)$ & $20{ }^{\circ} \mathrm{C}$ \\
\hline Far-field temperature $\left(T_{e}\right)$ & $102.5^{\circ} \mathrm{C}$ \\
\hline Other reservoir assumptions & $\begin{array}{l}\text { Laterally-extensive, homogeneous, } \\
\text { horizontally-isotropic }\end{array}$ \\
\hline
\end{tabular}

\subsection{Impedance Analytical Solutions}

When fluid is injected into one well and extracted from another, the reservoir impedance is the pressure difference between the two wells divided by the mass flow rate. All else equal, a low impedance will allow a higher flow rate, which likewise increases electric power production.

We analyze two well patterns in this work: a tessellated, developed field (TDF) and an isolated doublet (ID). The 5-spot pattern in Adams et al. [2015] is an example of a tessellated, developed field (TDF), which we define as a geothermal field that contains a repeating pattern of wells in a laterallyextensive, homogeneous and horizontally-isotropic reservoir. We assume reservoirs are homogeneous and horizontally-isotropic for the entirety of this study. When analyzing a TDF, we use symmetry to simplify boundary conditions and consider only a small section of the geothermal field. Then, the results from the small section can be scaled up to represent larger parts of the field.

While the TDF represents a mature field, many geothermal fields will start with a single injectionproduction pair. We define an isolated doublet (ID) as an injection-production well pair with non-zero well spacing located far from any other wells, so there is no outside hydraulic or thermal influence. Many geothermal fields will start as an ID and become a TDF as development continues. Therefore, we analyze IDs and TDFs, which act as bounding cases.

We provide several analytical solutions for reservoir impedance here: a square, 5-spot TDF well pattern in cartesian coordinates (Sec. 2.1.1), a concentric circle approximation of an ID (Sec. 2.1.2) in radial coordinates, and a more exact solution for an ID using a stream potential function (Sec. 2.1.3). In Sec. 2.1.4, we present a hybrid 5-spot TDF solution. This hybrid solution combines desirable aspects of cartesian and radial coordinate systems used in Secs. 2.1.1 and 2.1.3. Pressure contours for the ID and TDF approaches are illustrated in Fig. 2(a) and Fig. 2(b), respectively. These contour lines represent the cross-sectional area over which flow occurs divided by the reservoir thickness.

a)

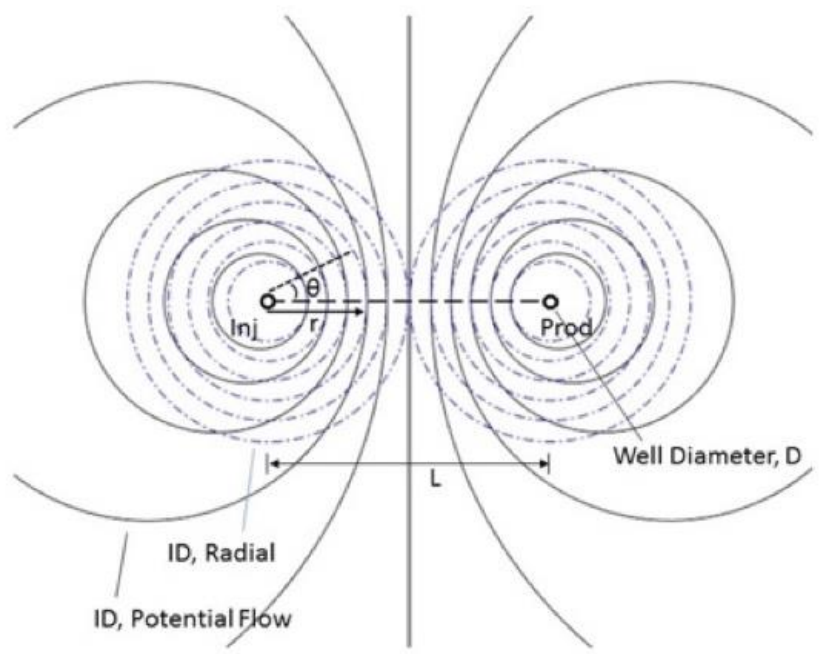

b)

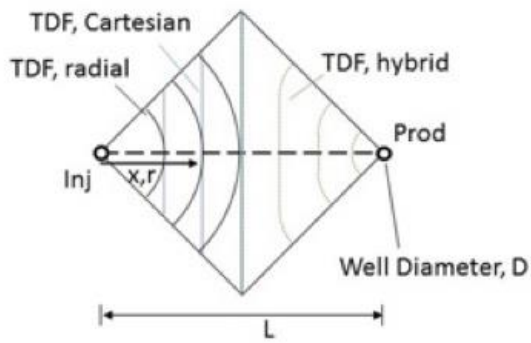


Fig. 1. Pressure contours for (a) ID and (b) one-quarter of a 5-spot TDF in plan view. These pressure contours correspond to the crossssectional area divided by the reservoir thickness for the solutions derived in Sec. 2.1.

\subsubsection{Tessellated, Developed Field (TDF) in Cartesian Coordinates}

The one-dimensional Darcy equation for horizontal flow can be expressed in Equation 1, where $P$ is the pressure, $\mu$ is the dynamic viscosity of the fluid, $V$ is the Darcy velocity, $\kappa$ is the permeability, and $x$ is distance in the direction of fluid flow.

$$
d P=-\frac{\mu \cdot V}{\kappa} d x
$$

The continuity equation is shown in Equation 2, where the mass flowrate, $\dot{m}$, is equal to the product of fluid density, $\rho$, cross-sectional area, $A_{c}$, and Darcy velocity, $V$.

$$
\dot{m}=\rho \cdot A_{c} \cdot V
$$

Equation 2 is substituted into Equation 1, yielding Equation 3.

$$
d P=-\frac{\mu}{\rho} \frac{\dot{m}}{\kappa \cdot A_{c}} d x
$$

For a one-quarter domain of a 5 -spot configuration, the $\mathrm{x}$-axis is aligned on the line between the injection and production wells (see Fig. 1(b)). The cross-sectional area, $A_{c}$, is given in Equation 4, where $b$ is the reservoir thickness. The lines "TDF, Cartesian" in Fig. 1(b) indicate the cross sectional area divided by the reservoir thickness.

$$
A_{c}=2 \cdot x \cdot b
$$

By combining Equations 3 and 4 and integrating from the interface between the well and the porous media, $x_{0}$, to the midpoint between the two wells, $L / 2$, we arrive at Equation 5 where $L$ is the distance between the injection and production wells (i.e., the well spacing). Integration of Equation 5 yields Equation 6, where $\Delta P$ is the pressure decrease from the well to the midpoint.

$$
\begin{gathered}
\int d P=-\frac{\bar{\mu}}{\bar{\rho}} \frac{\dot{m}}{2 \cdot \kappa \cdot b} \int_{x_{0}}^{\frac{L}{2}} \frac{1}{x} d x \\
\Delta P=\frac{\bar{\mu}}{\bar{\rho}} \frac{\dot{m}}{2 \cdot \kappa \cdot b} \ln \left(\frac{L}{2 \cdot x_{0}}\right)
\end{gathered}
$$

To find the total pressure difference from the injection well to the production well, $\Delta P_{\text {tot }}$, Equation 6 must be doubled. The viscosity, $\bar{\mu}$, and density, $\bar{\rho}$, are effective values within the entire reservoir. Also, the well perimeter in this one-dimensional Cartesian space is a square, where each side of the square has a length of $2 \cdot x_{0}$. Thus, it is possible to equate the perimeter of the square with the perimeter of a circle with diameter, $D$, to yield Equation 7 .

$$
\pi \cdot D=4 \cdot\left(2 \cdot x_{0}\right)
$$

Substitution of Equation 7 into Equation 6 yields the pressure difference as a function of well diameter, $D$. This equation is valid for all quadrants of the 5-spot configuration, and the total flow rate through the well is $\dot{m}_{t o t}=4 \dot{m}$, due to the symmetry of the problem. We express the impedance in terms of $\Delta P_{t o t}$ and $\dot{m}_{t o t}$ in Equation 8, which leads to easier comparison with other solutions in Sec 2.1. Note that the reservoir impedance is inversely proportional to the reservoir transmissivity, $\kappa b$, which is the product of reservoir permeability and thickness.

$$
\frac{\Delta P_{t o t}}{\dot{m}_{t o t}}=\frac{\bar{\mu}}{\bar{\rho}} \frac{1}{4 \cdot \kappa \cdot b} \ln \left(\frac{4 \cdot L}{\pi \cdot D}\right)
$$

\subsubsection{Isolated Doublet (ID) and Tessellated, Developed Field (TDF) in Radial Coordinates}

A similar approach as in Sec. 2.1.1 is used to solve for the reservoir impedance of an injectionproduction pair that is far from the influence of other wells (i.e., an ID). We assume the flow between the injection and production wells flows uniformly and radially in all directions, up to a radius of $L / 2$. 
For this injection-production pair, we use radial coordinates and substitute $d x=d r$ in Equation 1 . The cross-sectional area of flow is the product of the circumference at any given radius and the reservoir thickness, given in Equation 9. The cross sectional area divided by the reservoir thickness is illustrated by the lines labeled "ID, Radial" in Fig. 1(a).

$$
A_{c}=2 \cdot \pi \cdot r \cdot b
$$

By combining Equations 3 and 9, we obtain Equation 10, where $r_{0}$ is the well radius, which is analogous to $x_{0}$ from Sec. 2.1.1. Integration yields Equation 11 which is the pressure difference from the well to the midpoint.

$$
\begin{gathered}
\int d P=-\frac{\bar{\mu}}{\bar{\rho}} \frac{\dot{m}}{2 \cdot \pi \cdot \kappa \cdot b} \int_{r_{0}}^{\frac{L}{2}} \frac{1}{r} d r \\
\Delta P=\frac{\bar{\mu}}{\bar{\rho}} \frac{\dot{m}}{2 \cdot \pi \cdot \kappa \cdot b} \ln \left(\frac{L}{2 \cdot r_{0}}\right)
\end{gathered}
$$

Equation 11 is rearranged into the reservoir impedance of an approximate source-sink pair in Equation 12, where the well radius is half the well diameter. Note that the flow rate, $\dot{m}$, is equal to $\dot{m}_{\text {tot }}$ in this section because we analyzed the entire circumference of the well, unlike in Sec. 2.1.1 where we only analyzed one-quarter of the circumference.

$$
\frac{\Delta P_{t o t}}{\dot{m}_{t o t}}=\frac{\bar{\mu}}{\bar{\rho}} \frac{1}{\pi \cdot \kappa \cdot b} \ln \left(\frac{L}{D}\right)
$$

It turns out that the radial approach can also be applied to derive the impedance of a 5-spot TDF, and the impedance for "ID, Radial" are the same as "TDF, Radial" (i.e. Equation 12 also applies to a 5-spot TDF analyzed with radial coordinates). This can be derived following the same approach as in Sec. 2.1.1, except using the lower bound of integration in Equation 5 is $D / 2$ and $A_{c}=\pi r b / 2$. Since a similar derivation was already been shown, it is not repeated.

Note that for large values of $L / D$, the reservoir impedance of Equation 12 is larger than the reservoir impedance of a 5 -spot (Equation 8) by a factor of $4 / \pi$. This $27 \%$ difference is due to the $27 \%$ smaller swept area of the "ID, Radial" and "TDF, Radial" solutions than the "TDF, Cartesian" solution. Thus, in Sec. 2.1.3, we use the potential functions to include the entirety of the reservoir area for an ID.

\subsubsection{Isolated Doublet (ID) with Potential Flow}

The pressure potential curve surrounding a source (i.e., injection well) or sink (i.e., production well) does not have a constant radius, as is assumed in Sec 2.1.2. Thus, to gain a more precise solution for an ID, we integrate the distance of the potential curve surrounding the sink to obtain the cross-sectional area, given in Equation 13, where $r_{1}$ is the radius to the equipotential line from the sink and $\theta$ is the inclination.

$$
A_{c}=b \cdot \int_{0}^{2 \pi} r_{1} d \theta
$$

For source-sink potential flow, the stream potential, $\varphi$, is given by Equation 14, where $M$ is the source-sink strength, $r_{1}$ is the radial distance from the sink and $r_{2}$ is the radial distance from the source [Stern 2006].

$$
\varphi=-\frac{M}{2 \pi} \ln \left(\frac{r_{1}}{r_{2}}\right)
$$

On the shortest streamline which extends directly from the sink to source (shown as a dashed line in Fig. 1(a)), the potential is Equation 15, evaluated where $r_{1}=r$ and $r_{2}=L-r$ along this streamline.

$$
\varphi=-\frac{M}{2 \pi} \ln \frac{r}{L-r}
$$

Equating Equations 14 and 15, yields Equation 16, which provides the distances $r_{1}$ and $r_{2}$ along a line of constant potential as a function of $L$ and $r$. Equation 16 is solved for $r_{2}^{2}$ in Equation 17. 


$$
\begin{gathered}
\frac{r_{2}}{r_{1}}=\frac{L}{r}-1 \\
r_{2}^{2}=r_{1}^{2} \cdot\left(\frac{L}{r}-1\right)^{2}
\end{gathered}
$$

Separately of the considerations of potential flow, we find length $r_{2}$ as a function of $r_{1}$ and $\theta$ in Equation 18 using trigonometry.

$$
r_{2}^{2}=r_{1}^{2}+L^{2}-2 \cdot L \cdot r_{1} \cdot \cos \theta
$$

Equating Equations 17 and 18, solving for $r_{1}$, integrating according to Equation 13, and assuming large values of $L$ (i.e. $L \gg 1$ ) yields Equation 19.

$$
A_{c}=\frac{2 \cdot \pi \cdot b}{\left(\frac{1}{r^{2}}-\frac{2}{L \cdot r}\right)^{1 / 2}}
$$

The denominator in Equation 19 does not lend itself to simple integration. Thus, we make the approximation of Equation 20. This assumption preserves the anomaly occurring where $r=L / 2$ in which the cross-sectional area becomes infinite. At the midpoint between source and sink, the line of equal potential is tangent to streamline, and extends to infinity.

$$
\left(\frac{1}{r^{2}}-\frac{2}{L \cdot r}\right)^{1 / 2}=\left(\frac{1}{r}\right)^{1 / 2} \cdot\left(\frac{1}{r}-\frac{2}{L}\right)^{1 / 2} \approx\left(\frac{1}{r}-\frac{2}{L}\right)
$$

Equations 19 and 20 are combined with Equation 3 in radial space $(d x=d r)$ and integrated similar to Equation 10. The resulting reservoir impedance is given in Equation 21, where $e$ is Euler's number.

$$
\frac{\Delta P_{t o t}}{\dot{m}_{t o t}}=\frac{\bar{\mu}}{\bar{\rho}} \frac{1}{\pi \cdot \kappa \cdot b}\left[\ln \left(\frac{L}{D \cdot e}\right)+\frac{D}{L}\right]
$$

Equation 21, for the "ID, potential flow" solution is very similar to Equation 12, for the "ID, radial" solution, differing only by the factor of $e$ within the natural logarithm. The factor $D / L$ is comparatively small for large values of $L / D$ and can thus be neglected. For reservoir length to well diameter ratios of 1000 (i.e. $L / D=1000$ ), Equation 21 provides a reservoir impedance value approximately $15 \%$ smaller than Equation 12. The impedance value decreases to $22 \%$ for a ratio of 100 . This decreased impedance using the "ID, potential flow" method (Equation 21) is enabled by the infinite reservoir area, whereas the "ID, radial solution" (Equation 21) has a finite swept volume.

\subsubsection{Tessellated, Developed Field (TDF), Hybrid}

In a 5-spot TDF, the cross-sectional area divided by the reservoir thickness is best represented by a circular arc near the well, but it is best represented by a straight line at the midpoint. We define a hybrid cross-sectional area in Equation 22. The hybrid area divided by the reservoir thickness is illustrated in Fig. 1(b) by the lines "TDF Hybrid":

$$
A_{c}=\frac{2 b}{\frac{L}{2}-x_{o}}\left(x^{2}\left(1-\frac{\pi}{4}\right)+x\left(\frac{L \pi}{8}-x_{o}\right)\right)
$$

Otherwise, the impedance calculation follows the approach used in Sec. 2.1.1 and 2.1.2 to find the reservoir impedance:

$$
\frac{\Delta P_{\text {tot }}}{\dot{m}_{\text {tot }}}=\frac{\bar{\mu}}{\bar{\rho}} \frac{1}{\pi \cdot \kappa \cdot b} \ln \left(\frac{\pi}{4} \frac{L}{D}\right)
$$

Care should be used with all of the derivations from Sec. 2.1, as they are one-dimensional, rely on average fluid properties, and assume a uniform velocity profile through a cross-sectional area. Thus, they do not account for flow deviations, heterogeneities, or buoyancy. They do, at minimum, provide an orderof-magnitude approximation of the reservoir behavior for use within a geothermal system.

\subsection{Wellbore Heat Loss}

Heat loss to the rock surrounding the wellbore is implemented using a semi-analytical approach. In each wellbore element that is numerically integrated [Adams et al., 2015], an analytical heat solution for 
a semi-infinite solid is applied from Zhang et al., [2011]. This approach assumes: heat conduction only occurs radially to the far-field and the wellbore wall temperature is constant with time.

Similar to [Adams et al., 2015], the wellbore elements are evaluated numerically, with pressure (Equation 24) and energy (Equation 25) balances across each element, where $\Delta z$ is the change in element elevation, $\Delta P_{\text {Loss }}$ is the pipe frictional loss, $h$ is the enthalpy, $Q_{\text {Loss }}$ is the heat exchange to the surroundings, $\dot{m}$ is the fluid mass flowrate, and an "i" subscript indicates the value at the ith element. The pressure loss equation is identical to Adams et al. [2015], while the energy equation includes the new heat exchange term.

$$
\begin{gathered}
P_{i+1}=P_{i}-\rho_{i} \cdot g \cdot \Delta z-\Delta P_{\text {Loss }, i} \\
h_{i+1}=h_{i}-g \cdot \Delta z-\frac{Q_{\text {Loss }, i}}{\dot{m}}
\end{gathered}
$$

The heat loss is solved using Equation 26 from Zhang et al. [2011], where $k_{e f f}$ is the effective thermal conductivity of rock/water porous media mixture, $\beta$ is a non-dimensional, time-dependent factor for heat transfer, $T_{w}$ is the well casing temperature and $T_{e}$ is the background, far-field temperature at depth equal to the product of geologic temperature gradient and depth plus the average surface temperature. It is assumed that the well casing and fluid heat transfer resistances are small compared to conduction through the rock and are thus neglected.

$$
Q_{L o s s, i}=\Delta z \cdot 2 \cdot \pi \cdot k_{e f f} \cdot \beta \cdot\left(T_{w, i}-T_{e, i}\right)
$$

The wall temperature is an intermediate temperature between the wellbore fluid temperature and farfield temperature. Without any heat loss, the wellbore elements are assumed to be sufficiently long that the fluid temperature is in thermal equilibrium with the well wall. Thus, the well wall temperature, $T_{w}$, is assumed to be the resulting fluid temperature for an enthalpy of $h_{i}$ and pressure of $P_{i}$ if the heat loss term in Equation 25 were zero.

The dimensionless factor $\beta$ is given by Zhang et al. [2011] as Equation 27, where $t_{d}$ is the dimensionless time.

$$
\beta= \begin{cases}\left(\pi \cdot t_{d}\right)^{-1 / 2}+\frac{1}{2}-\frac{1}{4}\left(\frac{t_{d}}{\pi}\right)^{\frac{1}{2}}+\frac{1}{8} t_{d}, & t_{d} \leq 2.8 \\ \frac{2}{\ln \left(4 \cdot t_{d}\right)-1.16}-\frac{1.16}{\left(\ln \left(4 \cdot t_{d}\right)-1.16\right)^{2}}, & t_{d}>2.8\end{cases}
$$

The dimensionless time, $t_{d}$, is given in Equation 28, where $\omega$ is the thermal diffusivity of the rock/fluid porous media mixture and $t$ is the time.

$$
t_{d}=\omega \frac{4 \cdot t}{D^{2}}
$$

\subsection{Reservoir Heat Depletion}

Recent work used numerical solutions to show how reservoir heat depletion affects electric power output [Adams et al., 2020]. We adapt a heat depletion analytical solution from Carslaw and Jager [1986] to estimate the sedimentary basin heat depletion. This approach is similar to that used by Zhang et al. [2011].

The reservoir is treated as a sphere with infinite thermal conductivity, which is situated within a semiinfinite media at the background, far-field geothermal temperature. The temperature of the reservoir is [Carslaw and Jager, 1986]:

$$
T_{r}=\frac{-Q}{4 \pi a_{A} k_{e f f}} \epsilon(t)+T_{e}
$$

where $Q$ is the rate of heat removal from the reservoir, $a$ is the equivalent radius of the reservoir and its subscript is discussed in more detail later in this section, and $\epsilon$ is a function of time and parameters related to reservoir size, geometry, and thermal diffusivity.

The rate of heat removal from a reservoir is: 


$$
Q=-\dot{m} c_{\text {Fluid }}\left(T_{\text {inj }}-T_{r}\right)
$$

where $T_{i n j}$ is the temperature of the injection fluid and $c_{\text {Fluid }}$ is the working fluid's heat capacity. We assume that that heat extraction rate is constant (to be consistent with Equation 29) and we substitute Equation 30 into Equation 29. While $Q$ will actually decrease with time as the reservoir temperature decreases (assuming a constant mass flowrate), Equation 29 will give approximate values of reservoir temperature at early time, before the temperature declines are substantial. We are most interested in temperature depletion at these early times. For later times, when the temperature depletion is large, the solution to Equation 29 will be conservative and over predict temperature depletion.

With algebraic manipulation of Equations 29 and 30, a non-dimensional reservoir temperature is expressed as:

$$
\Gamma=\frac{T_{r}-T_{i n j}}{T_{e}-T_{i n j}}=\left(\frac{\dot{m} c_{F l u i d} \epsilon}{4 \pi a_{A} k_{e f f}}+1\right)^{-1}
$$

And $\epsilon$ is defined as:

$$
\epsilon=\frac{1+a_{V} \lambda}{a_{V} \lambda}-\frac{2 a_{V}^{2} \xi^{2} \lambda^{2}}{\pi} \int_{0}^{\infty} \frac{\exp \left(\frac{\omega u^{2} t}{a_{V}^{2}}\right) d u}{\left[\left(u^{2}\left(1+a_{V} \lambda\right)-\xi a_{V} \lambda\right)\right]^{2}+\left[u^{3}-\xi a_{V} \lambda u\right]^{2}}
$$

where $\lambda$ is the inverse of the product of the contact resistance between the reservoir and surrounding rock/fluid mixture and the thermal conductivity of the surrounding rock/fluid mixture, $\xi$ has a value of three when the density and heat capacity of the reservoir are the same as the surrounding rock (as we assume), and $u$ is a variable of integration.

As an aside, we note that the non-dimensional temperature in the absence of conduction is:

$$
\Gamma=\frac{T_{r}-T_{i n j}}{T_{e}-T_{\text {inj }}}=\left(\frac{t \dot{m} c_{\text {Fluid }}}{V_{\text {res }} \rho_{\text {eff }} c_{\text {eff }}}+1\right)^{-1}
$$

where $V_{\text {res }}$ is the reservoir volume.

In the limit as $\lambda$ approaches infinity, $\epsilon$ can be expressed more simply as:

$$
\epsilon=1-\frac{2 \xi^{2}}{\pi} \int_{0}^{\infty} \frac{\exp \left(\frac{\omega u^{2} t}{a_{V}^{2}}\right) d u}{\left(u^{2}-\xi\right)^{2}+(\xi u)^{2}}
$$

Analytical solutions of $\epsilon$ exist for both early and late time [Carslaw and Jager, 1986], but we solve the integral in Equation 34 numerically so that the solution covers all timeframes that are relevant for geothermal development, including intermediate times that the analytical solutions do not represent well.

In the original Carslaw and Jager [1986] solution, $a$ represents the radius of the sphere. However, since geothermal reservoirs are not typically spherical, we found that careful specification of the equivalent radius can improve the solution. The equivalent radius could be specified to match the reservoir volume, $a_{V}$, or the surface area over which conduction occurs, $a_{A}$ :

$$
\begin{gathered}
a_{V}=\left(\frac{3 V_{\text {res }}}{4 \pi}\right)^{\frac{1}{3}} \\
a_{A}=\sqrt{\frac{A_{\text {res }}}{4 \pi}}
\end{gathered}
$$

where $A_{\text {res }}$ is the surface area of the reservoir exposed to conduction from the surroundings.

In offline comparisons to the numerical solution for reservoir heat depletion (see Sec. 2.4), we found that it is best to use the area-based equivalent radius outside of $\epsilon$ (i.e., in Equations 29 and 31). This ensures that the temperature is correct as the time approaches infinity because the late-time steady state temperature occurs when the heat extraction rate is equal to the rate of conductive heat flow into the reservoir. We use the volume-based radius within the calculation of $\epsilon$ (Equation 34), which gives the reservoir the right initial amount of heat. 


\subsection{Numerical Simulation}

We also perform thermo-hydraulic reservoir simulations for comparison to the analytical solutions. The simulations calculate reservoir impedance, which is necessary to estimate electric power generation. The simulations also calculate the temperature at the production well with respect to time.

The simulations are performed using the reservoir simulator TOUGH 2 and investigate one-eighth of a five-spot TDF, similar to Adams et al. [2015]. The input file and mesh (included in the Supplemental Information) are based on an example in the TOUGH2 manual and were intentionally changed as little as possible. The mesh contains 37 elements, consistent with the TOUGH 2 manual example and previous studies [Adams et al., 2015; Randolph and Saar, 2011]. We apply fixed mass flow rate boundary conditions at the injection and production cells. We maintain the pressure at the corner node as the hydrostatic reservoir pressure by supplying constant pressure fluid as needed. Due to symmetry, there is no flux of heat or fluid across the vertical boundaries. There is also no fluid flux through the top or bottom boundaries because the overlying and underlying rock are assumed to be impermeable. Heat is conducted into the reservoir from the top and bottom boundaries according to a built-in TOUGH2 analytical functionality, which assumes Cartesian, one-dimensional heat conduction coming from the background geothermal temperature $\left(T_{e}\right)$, which acts as a local thermal source term to the numerical solution on the grid. The working fluid is water.

In Sec. 3.4, we also present results for a thin reservoir with a thickness of $30 \mathrm{~m}$, one-tenth the base case's thickness.

\subsection{Estimating Electricity Generation}

The electrical power generation is estimated with a similar approach to Adams et al. [2015] for the indirect water system (see Fig. 2), with two changes. First, the TOUGH2 reservoir simulator is replaced with an analytical solution for impedance (see Sec 2.1). Second, wellbore heat loss is included for the production well (see Sec. 2.2), which was not considered in Adams et al. [2015]. Power generation is found without considering pressure depletion in the reservoir.

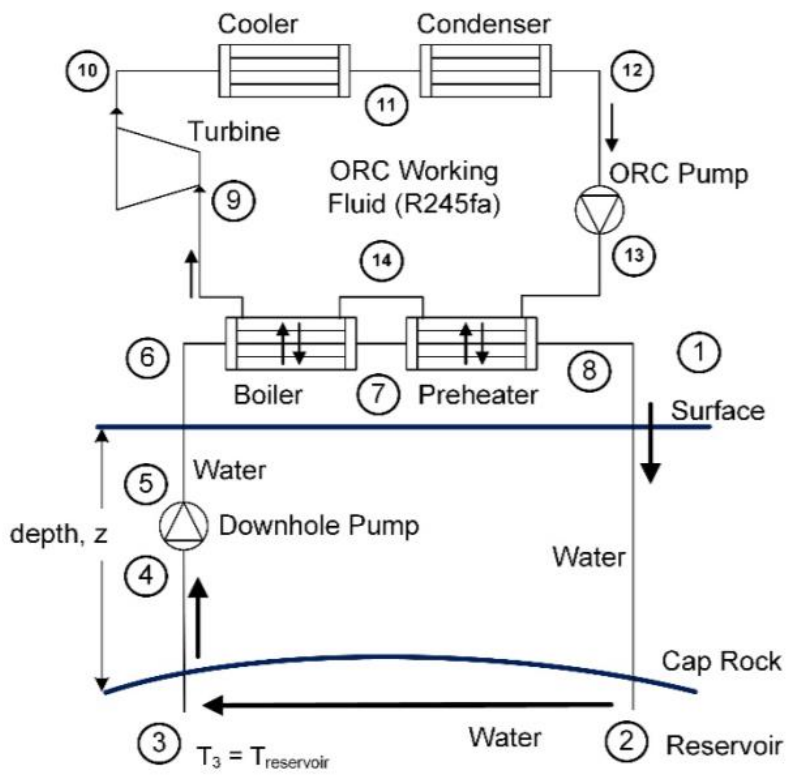

Fig. 2. Cycle schematics for an indirect subsurface-water Organic Rankine Cycle (ORC) geothermal power plant. Modified from Adams et al. (2015).

Fig. 2 shows the cycle schematics for the indirect water Organic Rankine Cycle (ORC). In the lower loop, the primary fluid (water) is circulated from the surface; through the reservoir, where it gains heat; and back to the surface, where it gives heat to the secondary working fluid. In the upper loop, the secondary fluid (R245fa) is heated from the primary fluid and drives the ORC to produce electricity. For more description of the ORC, see Adams et al. [2015].

\section{Results and Discussion}

In much of Sec. 2, we derived equations for a 5-spot within a tessellated, developed field (TDF). In Sec. 3.1, we show that, for the assumptions employed, the subsurface response for a 5-spot TDF is 
identical to that of a doublet TDF, and therefore equations for a 5-spot TDF apply equally to a doublet TDF. In Sec. 3.2, we summarize the analytical reservoir impedance solutions and compare them to the numerical solution. In Sec. 3.3, we use the reservoir impedance from Sec. 2.1, the wellbore heat loss from Sec. 2.2, and the power cycle model to calculate the initial electric power generation. In Sec. 3.4, we compare reservoir heat depletion results.

\subsection{Similarities Between 5-Spot and Doublet Tessellated, Developed Fields}

Fig. 3 shows a well pattern in plan view. The black dashed line indicates the boundaries of one unit of a 5-spot TDF, while the blue solid line shows the boundaries of one unit of a doublet TDF. The fivespot TDF has a power plant located at the injection well and takes one-fourth of the fluid from each of the four neighboring production wells. In contrast, the doublet TDF has a power plant located between the injection and production wells and circulates flow only between these two wells.

We infer from Fig. 3 that the only difference between the two TDF setups is the surface location of the power plants and piping, for the assumptions (e.g., a homogeneous, horizontal-isotropic, laterallyextensive reservoir) utilized in this study. Therefore, we conclude that the reservoir impedance and the thermal recovery will be the same in a TDF full of five-spots as a TDF full of doublets, and the analysis of a five-spot TDF applies equally to a doublet TDF. We do not distinguish between a 5-spot TDF and a doublet TDF for the rest of the paper. From a practical standpoint, it is advantageous to build surface infrastructure as a TDF doublet pattern rather than a TDF 5-spot pattern because it reduces the amount of piping to bring fluid to and from the powerplants. The piping required for one unit of a doublet TDF has an approximate length of $L$, whereas the piping needed for one unit of a 5-spot TDF has an approximate length of $4 L$, as can be seen in Fig. 3.

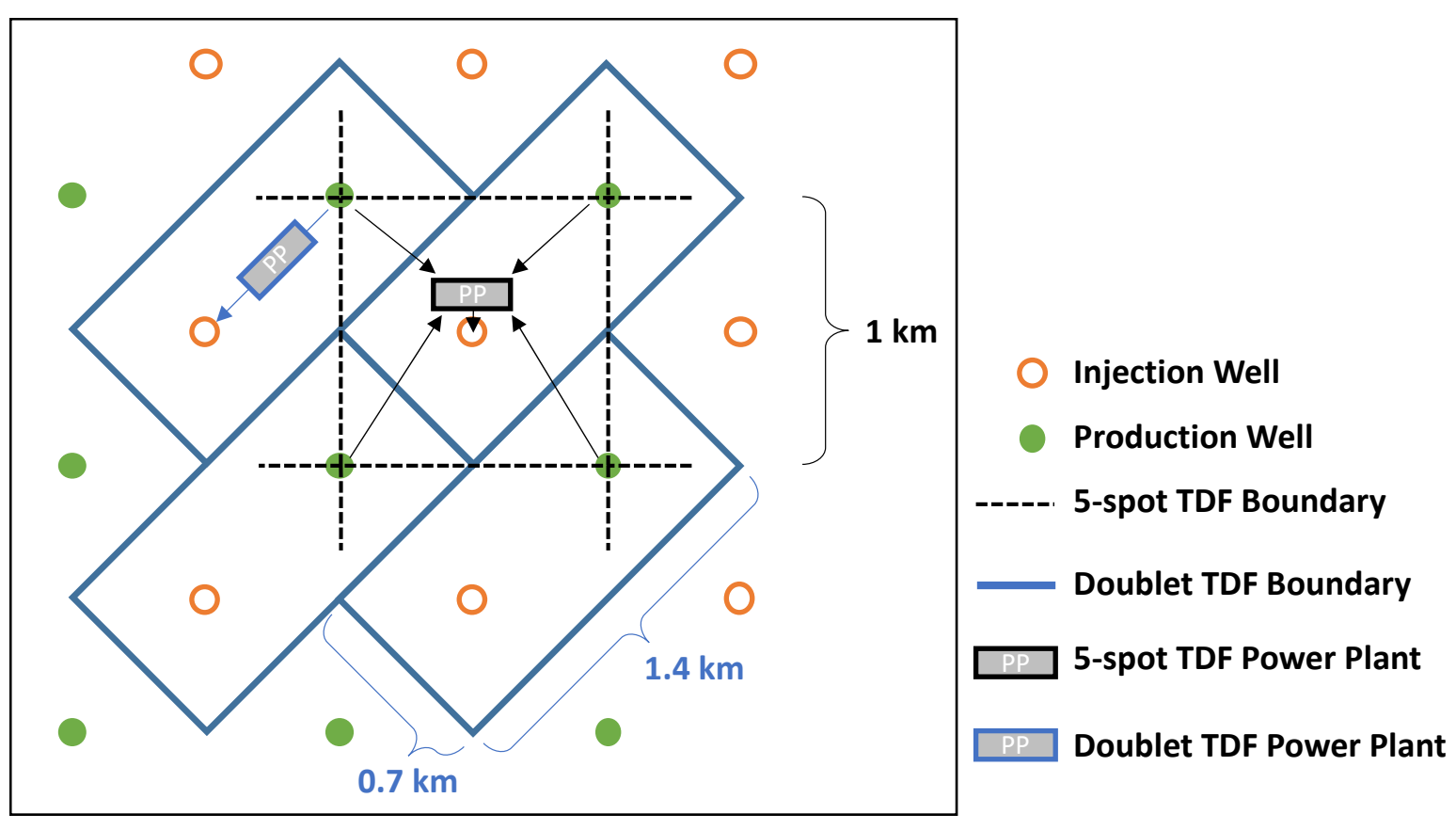

Fig. 3. Plan-view comparison of 5-spot TDF and doublet TDF. The surface infrasturcutre is different, but the subsurface response is identical.

\subsection{Reservoir Impedance}

We find that the reservoir impedance solutions from Sec. 2.1 can be expressed more concisely. The solutions can be calculated generally as a function of the location of the well/porous media interface (i.e., $x_{0}$ or $\left.r_{0}\right)$ and the cross-sectional area $\left(A_{c}\right)$ and then expressed in terms of constants $C_{1}$ and $C_{2}$ :

$$
\frac{\Delta P_{t o t}}{\dot{m}_{t o t}}=\frac{2 \bar{\mu}}{\bar{\rho}} \frac{1}{\kappa} \int_{x_{o}}^{\frac{L}{2}} \frac{d x}{A_{c}(x)}=\frac{\bar{\mu}}{\bar{\rho}} \frac{1}{\kappa b} C_{1} \ln C_{2}
$$

The solutions of Sec. 2.1 are summarized in Table II using the variables $A_{c}, C_{1}, C_{2}$, and $x_{0}$ or $r_{0}$ from Equation 37. The final column shows the value of $C_{1} \ln C_{2}$, which is proportional to the impedance, with an assumed value of $L / D=1000$. Notably, the differences in impedance are small amongst all 
analytical solutions (the range for $C_{1} \ln C_{2}$ is 1.8 to 2.2 ), which indicates that the ID does not have a significantly different impedance than the TDF. It is also notable that the TDF hybrid solution has a value between the TDF radial solution and the TDF cartesian solution. We used the TDF hybrid solution moving forward because it honors the radial flow near the well and the linear flow near $x=L / 2$, as discussed in Sec. 2.1.4. We use the "ID, potential" solution for the ID cases because it includes all the swept area.

TABLE II. ANALYTICAL IMPEDANCE. RIGHT COLUMN INDICATES VALUE FOR $L=1000 \mathrm{D}$.

\begin{tabular}{|c|c|c|c|c|c|}
\hline Well Pattern & $x_{0}$ or $\boldsymbol{r}_{0}$ & $A_{c}$ & $C_{1}$ & $C_{2}$ & $C_{1} \ln \left(C_{2}\right)$ \\
\hline ID, Radial (Sec. 2.1.2) & $\frac{D}{2}$ & $2 \pi r b$ & $\frac{1}{\pi}$ & $\frac{L}{D}$ & 2.2 \\
\hline ID, Potential Flow (Sec. 2.1.3) & $\frac{D}{2}$ & $\frac{2 \cdot \pi \cdot b}{\left(\frac{1}{r^{2}}-\frac{2}{L \cdot r}\right)^{1 / 2}}$ & $\frac{1}{\pi}$ & $\frac{L}{D e}$ & 1.9 \\
\hline TDF, Cartesian (Sec. 2.1.1) & $\frac{\pi D}{8}$ & $2 x b$ & $\frac{1}{4}$ & $\frac{4}{\pi} \frac{L}{D}$ & 1.8 \\
\hline TDF, Radial (Sec. 2.1.2) & $\frac{D}{2}$ & $\frac{\pi r b}{2}$ & $\frac{1}{\pi}$ & $\frac{L}{D}$ & 2.2 \\
\hline TDF, Hybrid (Sec. 2.1.4) & $\frac{D}{2}$ & $\frac{2 b}{\frac{L}{2}-x_{o}}\left(x^{2}\left(1-\frac{\pi}{4}\right)+x\left(\frac{L \pi}{8}-x_{o}\right)\right)$ & $\frac{1}{\pi}$ & $\frac{\pi}{4} \frac{L}{D}$ & 2.1 \\
\hline
\end{tabular}

We compare the impedance as a function of time for the numerical and two analytical solutions in Fig. 4. The analytical solutions represent the most-accurate TDF and ID solutions (i.e., the "ID, streamlines" and "TDF, hybrid" entries from Table II). Fluid properties are pressure- and temperaturedependent, which is accounted for internally in TOUGH2. For the analytical solution, the temperature from Equation 31 and the hydrostatic pressure are inputs to the CoolProp [Bell et al., 2014] python library, which calculates the fluid properties.

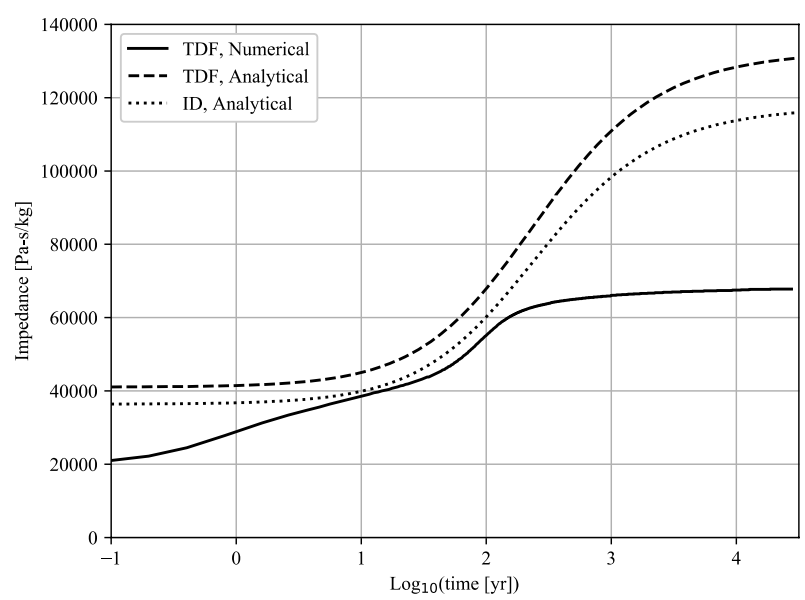

Fig. 4. The TDF numerical solution uses TOUGH2. The TDF analytical solution uses the hybrid approach. The ID analytical solution uses the stream lines approach.

In all three solutions, the impedance increases with time due to the increase in water's kinematic viscosity as the reservoir cools, although the shape of the impedance curves differ because of to the way the viscosity is calculated. In the analytical solution, the kinematic viscosity varies both spatially and temporally as the reservoir cools. Since the region near the injection well starts cooling almost immediately, the kinematic viscosity near the injection well also increases at early times. The impedance increases steadily throughout time as the cooled region grows, and the impedance doubles by the tenth year of operation. In contrast, the analytical solution uses the average reservoir temperature and pressure to calculate the average kinematic viscosity of the reservoir, which does not account for spatial variations in fluid properties. It takes approximately ten years for the average temperature to change by $10 \%$ (see Sec 3.4), and therefore the average kinematic viscosity and the average impedance only increase by approximately $10 \%$ after ten years. However, as the reservoir continues to cool, the impedance continues to increase, doubling after approximately 200 years. 
The numerical solution gives a lower impedance than either of the analytical solutions, which are similar to each other, differing by only $13 \%$. At early and late times when the fluid properties are in agreement, the analytical solutions calculate reservoir impedance roughly twice the numerical solution. Ravilov [2019] found that reservoir impedance increases with the number of nodes. Thus, the numerical solution in Fig. 4, with its relatively few nodes, likely provides a lower bound for the reservoir impedance. It is possible that the early-and late-time numerical solution would converge to the analytical solution with finer and finer discretization.

The Ravilov [2019] study raises an important consideration: the mesh discretization can have a large effect on reservoir impedance in numerical simulation. As the number of nodes increased from 1300 to 600,000 , the reservoir impedance increased from 38 to $48 \mathrm{kPa}-\mathrm{s} / \mathrm{kg}$. Through extrapolation, Ravilov [2019] predicted that an infinite number of nodes would lead to a reservoir impedance of $58 \mathrm{kPa}-\mathrm{s} / \mathrm{kg}$. It is difficult to know if the impedance asymptotes to a fixed value as the number of nodes goes to infinity. If this asymptote does exist, it is also difficult to know what its value of impedance will be. Ravilov's [2019] work suggests that getting an accurate reservoir impedance value using a numerical simulator requires nearly infinite computational resources.

It is difficult to say if the analytical or numerical impedance solutions are better. Each has its strengths and weaknesses and may be useful depending on the problem of interest. The analytical solution has a smaller computational expense than the numerical solution. On the other hand, the analytical solution also relies on simplifications, such as the use of average fluid properties, a one-dimensional coordinate system, and a uniform flow field. The numerical simulation does not rely on these simplifications, but it does depend on mesh discretization, as discussed in the previous paragraph. At the end of the day, the numerical and analytical solutions both give estimates of impedance; while we do not know which is better, we assume they represent a reasonable range.

\subsection{Electric Power Generation}

Fig. 5 shows the reservoir impedance and electric power generation as a function of transmissivity for various assumptions about reservoir impedance and wellbore heat exchange. These results follow the approach in Adams et al. [2015], except they include wellbore heat loss (from Sec. 2.2) and use the analytical solution for impedance (from Sec. 2.1) instead of the numerical solution. 
(a)

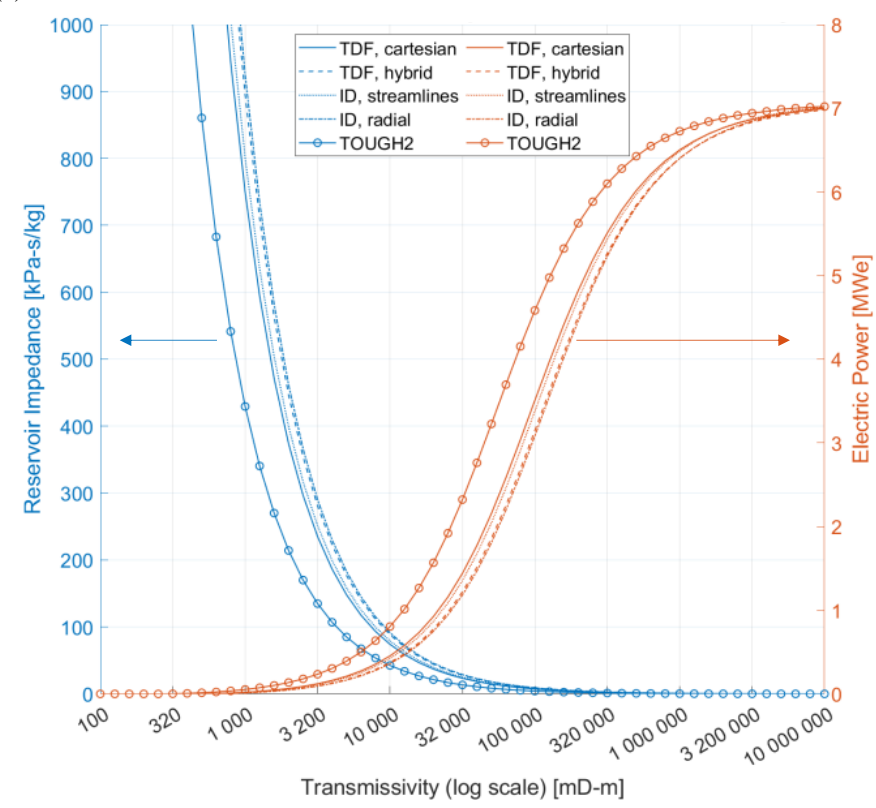

(b)

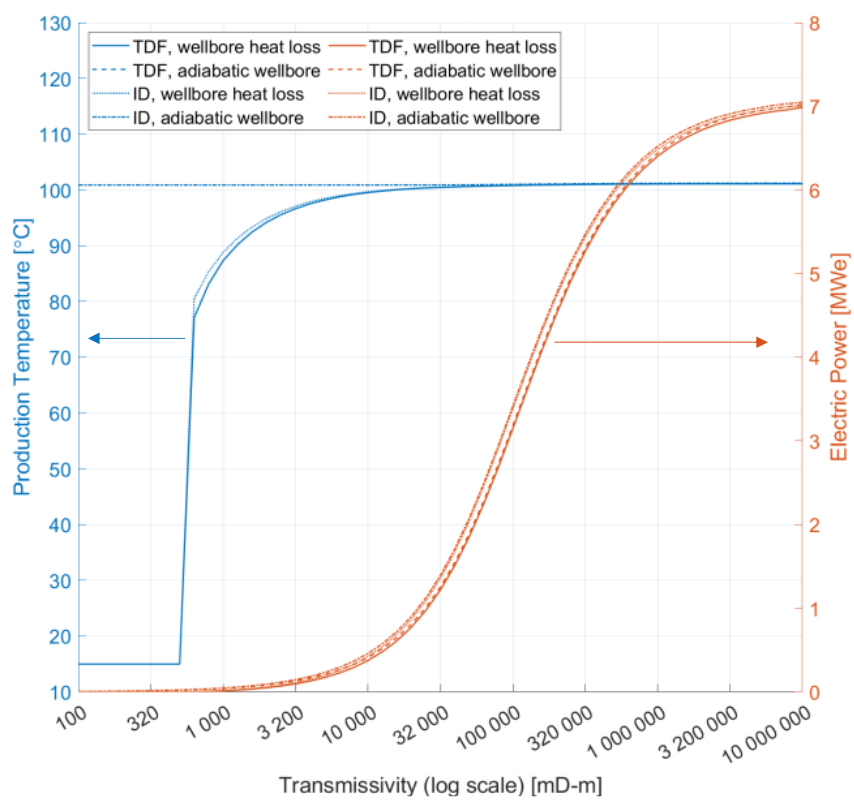

Fig. 5. Plot (a) illustrates the (in)sensitivity of power generation to the impedance model utilized. Plot (b) shows the (in)sensitivity of power generation to assumptions about wellbore heat loss. Both plots show four analytical impedance models, and (a) also shows the numerical impedance model. Horizontal arrows associate the curve to the relevant y-axis.

Fig. 5(a) shows the electric power generation as a function of transmissivity for various impedance models. There is little difference in the electric power generation $(<10 \%)$ between the four analytical impedance models because the differences in impedance among the analytical solutions are small $(\leq 18 \%)$. However, the numerical impedance in our model is roughly half of the analytical impedance, resulting in up to twice the electric power generation. As was stated in Sec. 3.2, the numerical and analytical solutions represent a range of impedances, and it is unclear which is better. In some applications, the uncertainty in the reservoir transmissivity may be much larger than the uncertainty introduced by the reservoir impedance model. Therefore, to calculate electric power generation accurately, it is crucial to reduce uncertainty in the reservoir transmissivity. However, there will likely always be some uncertainty in the prediction of electric power generation because it is difficult to reduce the uncertainty introduced by the reservoir impedance model to zero. 
Fig. 5(b) compares the solution with wellbore heat loss (Sec. 2.2) to an adiabatic solution to investigate the influence of wellbore heat loss on electrical power generation. At high transmissivity $(\geq$ $32,000 \mathrm{mD}-\mathrm{m}$ ), the production temperature is almost the same with and without heat loss (i.e., $\Gamma>0.98$ with heat loss and $\Gamma=1.0$ without heat loss), which is in agreement with previous work [Randolph and Saar, 2012]. The high transmissivity allow high flow rate, which allow the temperature to stay high [Ramey, 1962]. Namely, heat is flowing through the well at a high rate, and the conductive heat losses are small enough that they cannot significantly reduce the fluid temperature. The small difference in temperature between the heat-loss and adiabatic cases coincides with a small difference in electric power generation, which is different by $<4 \%$ for transmissivity $>32,000 \mathrm{mD}-\mathrm{m}$. The flow rate is small for low transmissivity $(<3,200 \mathrm{mD}-\mathrm{m})$, which results in power generation less than $0.2 \mathrm{MWe}$. At these low transmissivities (and flow rates), the production temperature is different between the adiabatic and heatloss cases, but the absolute difference that it causes in electric power generation is small. For intermediate transmissivity in the range of $3200-32,000 \mathrm{mD}-\mathrm{m}$, wellbore heat losses seem to be most relevant for power generation. The flow rate in this range of transmissivity is low enough that wellbore heat losses decrease the temperature while still large enough that a significant amount of electric power is generated. However, even in this "sensitive" range, the effect of heat loss on power production is somewhat modest. At $10,000 \mathrm{mD}-\mathrm{m}$, the adiabatic approach predicts $<0.1 \mathrm{MWe}$ more power production, which is a difference $<30 \%$ compared to the approach with heat loss.

\subsection{Reservoir Heat Depletion}

As the temperature of a reservoir reduces, so does power generation. Therefore, understanding the reservoir temperature as a function of time offers insights for power generation as a function of time and reservoir lifetime.

Fig. 6 shows the non-dimensional reservoir temperature versus time for both the analytical and numerical solutions, which are calculated, respectively, from Equation 31 and in a post-processing step from the numerical solution. The solutions that neglect conduction (dotted lines in Fig. 6) are calculated from Equation 33. Subplot (a) uses the logarithm of time to illustrate late-time behavior, while (b) uses a linear scale to emphasize the shape of the curves at early times and the (un)importance of heat conduction to the reservoir temperature.
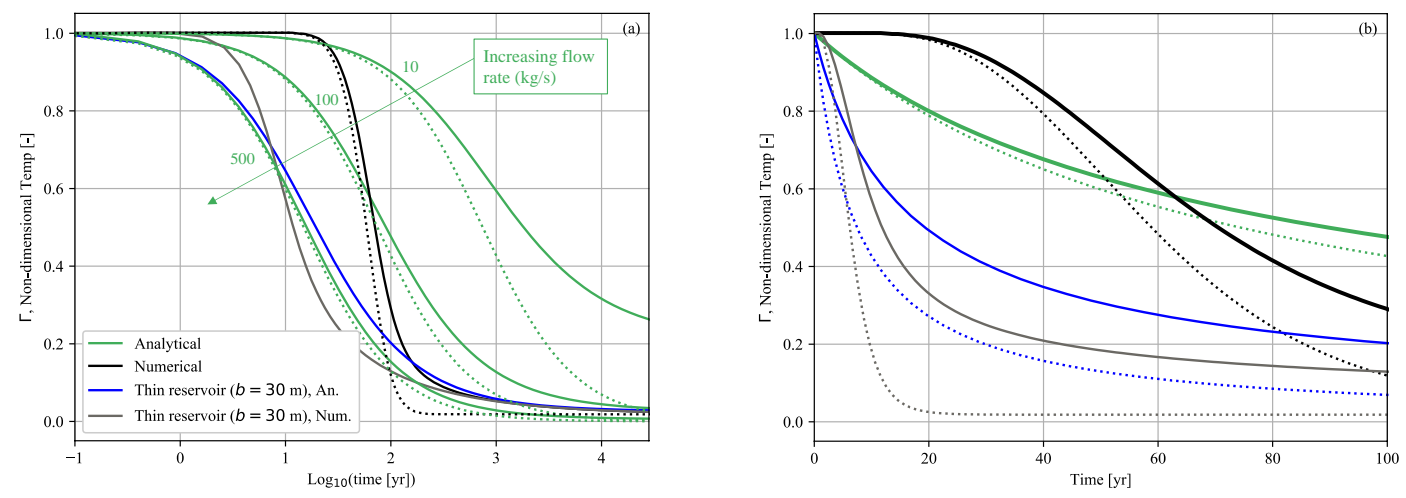

Fig. 6. The normalized reservoir temperature versus (a) logarithm of time and (b) time. In (a), mass flow rates are indicated for the three green, analtyical curves. All other curves use $100 \mathrm{~kg} / \mathrm{s}$. Dotted lines indicate scenarios that do not include heat conduction from the surroundings to the resrvoir, following the same color scheme.

\subsubsection{Comparison of Solutions}

The shape of the analytical and numerical solutions is different because they represent the temperature at different locations in the reservoir. The analytical solution shows the average temperature of the reservoir, which decreases at a decreasing rate at all times. In contrast, the numerical solution shows the production well's temperature, which decreases at an increasing rate until the thermal front reaches the production well. Then the temperature continues to decrease, but at a decreasing rate. This temperature versus time pattern in the numerical solution is a classic example of thermal breakthrough. It seems that both solutions are valid, but they measure different aspects of the temperature.

Another difference comes in the way that heat is removed from the reservoir. In the analytical solution, heat is removed at a constant rate. In the numerical solution, a flow rate and injection 
temperature are specified such that the initial heat removal rate matches the rate in the analytical solution. However, as the reservoir cools, the heat removal rate decreases. The numerical solution may be more realistic for sedimentary basin geothermal systems in this aspect, but the analytical solution represents a worst-case (i.e., cold) bound for heat depletion, especially at later times.

Other differences between the numerical and analytical solutions may be due to differences in problem geometry. The numerical solution uses a 3D geometry with $1 \mathrm{D}$, cartesian heat conduction from above and below the reservoir. In contrast, the analytical solution follows a 1D radial geometry, and the equivalent radius is adjusted to match the volume and surface area of the numerical solution (see Sec. 2.3).

Despite the differences discussed in the previous three paragraphs, both the analytical and numerical solutions describe heat depletion from a reservoir with the same volume and amount of removable heat. Conduction into the reservoir from the outsides is treated similarly in that they both use 1D analytical approaches. (Recall from Sec. 2.4 that in the numerical simulator the conduction into the reservoir is an analytical solution that is built into TOUGH2). The average temperature over the first 100 years is approximately the same for the base-case analytical and numerical solution, and the temperatures approach the same late-time steady state. Thus, it is possible to get an estimate of thermal depletion using both solutions.

The analytical solution could be used in at least two ways. Firstly, it can be interpreted as the average reservoir temperature. Under this approach, it provides a lower-bound on the reservoir temperature at all times due to its assumed constant rate of heat removal, as discussed earlier in this section. Secondly, the analytical solution could be interpreted as the temperature used for power production. Under this interpretation, the analytical solution is conservative at early times because it predicts a temperature lower than the production temperature from the numerical solution. The analytical solution is a computationally-cheap, lower-bound.

In many cases, the numerical solution may be more useful for power generation calculations, but it is useful to have the analytical solution as a computationally-cheap, lower-bound for use in power generation calculations, especially if the alternative is to neglect heat depletion as many studies have [Randolph and Saar, 2011; Adams et al. 2015].

\subsubsection{Physical Insights on Heat Depletion and Conduction}

Fig. 6(a) shows different flow rates and their effect on the analytical solution (see three green curves). For high flow rates, the temperature decreases more quickly. At late times, the temperature approaches a steady state greater than $T_{i n j}$ because conduction brings heat into the system at the same rate the working fluid removes it. This steady state is achieved at earlier times and lower temperatures for higher flow rates. In contrast, the no-conduction analytical solutions all approach $T_{i n j}$ at a late time because there is no heat recharge.

Fig. 6(b) compares the base case to a thin reservoir, highlighting that reservoir size and aspect ratio affect how long before thermal depletion occurs and how important conduction is. The base-case reservoir has a large volume, and therefore holds a lot of heat initially, which takes a long time to deplete, even if no conduction occurs. It takes 20 and 44 years, respectively, for the average temperature and the production temperature to deplete by $20 \%$. Neglecting conduction makes little difference to these results; the times for the average and production temperature to deplete by $20 \%$ are reduced by only $7 \%$ and $10 \%$, respectively. On the other hand, the thinner reservoir holds less heat initially, and the average and production temperature deplete by $20 \%$ after only 4 and 6 years, respectively. Conduction seems to play a relatively larger role for the thin reservoir. If conduction is neglected, the time for the average and production temperature to deplete by $20 \%$ are reduced by $60 \%$ and $40 \%$, respectively.

It seems that conduction matters more for a reservoir with high surface area to volume ratio. However, in our example, the conduction is similar for the base-case and thin reservoir scenarios because the surface area is the same. Perhaps the initial amount of heat in the reservoir (which is related to the reservoir volume) is more critical than conduction for avoiding early temperature depletion. It is prudent to consider if the reservoir's initial amount of energy will last for the geothermal project's proposed lifetime.

Conduction matters less for reservoirs with higher flow rates, as shown in the analytical solutions in Fig. 6(a). It takes $30 \%$ less time for the average temperature without conduction to reach $20 \%$ 
temperature depletion, compared to the solution with conduction. For $500 \mathrm{~kg} / \mathrm{s}$, the same difference is only $3 \%$. High flow rates are common in geothermal power production. For example, a recently-built geothermal project has $250 \mathrm{~kg} / \mathrm{s}$ flow rates per well [Adams et al., 2020]. Under these high-flowrates, the reservoir temperature is less sensitive to conduction, and having enough energy (related to reservoir volume) in the reservoir to last for the proposed lifetime is again worth considering.

\section{Conclusions}

This paper introduced and used analytical solutions to approximate reservoir impedance, wellbore heat loss, and reservoir heat depletion. The reservoir impedance and wellbore heat loss solutions were coupled to a power cycle model to calculate the electric power generation. The reservoir heat depletion solution gave insights about power generation decline over the reservoir lifetime. There are pros and cons to using analytical and numerical reservoir models. A careful weighing of objectives should be made when constructing a geothermal power plant model that couples reservoir performance with electricity generation. Some of the conclusions below can provide guidance when weighing these objectives. Others offer insights into the behavior of geothermal reservoirs and power production.

1. Under the assumptions in this paper (e.g., homogeneous, laterally-extensive reservoir), there is no difference in the subsurface between a TDF of 5-spots and a TDF of doublets. Therefore, these can both be treated as a TDF, and the equations for a 5-spot TDF also apply to a doublet TDF. Furthermore, the doublet pattern can potentially reduce the amount of surface piping compared to the 5-spot TDF pattern.

2. We cannot know whether the analytical or numerical impedance solution is better. The numerical solution gave a lower impedance than the analytical solutions, by approximately onehalf, which resulted in more power generation by up to a factor of two. Amongst the analytical solutions, we recommend the use of the "ID, Potential Flow" solution (Equation 21) for IDs and the "TDF, Hybrid" solution (Equation 23) for TDFs. The analytical solution utilizes simplifications (e.g., average fluid properties and uniform velocity profile through the crosssectional area). However, the analytical solution also has several benefits: (a) it is independent of grid resolution, (b) it is computationally inexpensive, and (c) it is probably easier to use than the numerical simulator for most people.

3. Wellbore heat losses may be justifiably neglected for small and large transmissivity (see Fig. $5(b)$ ). For the reservoir we investigated, wellbore heat loss had a moderate effect on power generation for transmissivities between 3,200 mD-m and 32,000 mD-m. Below 3,200 mD-m, the flow rate was low, which resulted in little electricity generation $(<0.2 \mathrm{MWe})$, with or without wellbore heat loss. Above $32,000 \mathrm{mD}-\mathrm{m}$, the flow rate was large enough that the production temperature depletion was insignificant (i.e., $\Gamma>0.98$ ), and the power generation was very similar with and without wellbore heat loss.

4. The reservoir heat depletion analytical and numerical solutions measure temperatures at different regions of the reservoir, but fundamentally they both reflect the temperature of reservoirs with the same initial amount of removable heat, which is replenished by conduction from the surroundings. It seems the analytical solution (Equation 31) represents a lower bound on the average reservoir temperature for all positive time and represents a lower bound on the production temperature in early years (see Sec. 3.4).

5. The amount of heat initially in the reservoir is closely related to the reservoir size and can be approximated from a lumped mass, with (Equation 31) or without (Equation 33) conduction. The thin reservoir reached $20 \%$ heat depletion faster than the thick reservoir. For the thin reservoir, all approaches (analytical and numerical with and without conduction) predicted $<10$ years before $20 \%$ heat depletion, whereas all approaches predict $>20$ years before $20 \%$ depletion for the thicker, base-case reservoir.

6. Reservoir temperature is more sensitive to conduction from the surroundings in cases with low flow rates (see Fig. 6(a)) or a high ratio of surface area to volume (e.g., the thin reservoir in Sec. 3.4). 


\section{Author Contributions}

DTB provided conceptualization, methodology, software, validation, visualization, and writing original draft. BMA provided conceptualization, methodology, software, validation, visualization, and writing - original draft. JOH provided writing - original draft and writing - review \& editing. MRF provided writing - review \& editing. JMB provided writing - review \& editing. MOS provided funding acquisition and writing - review \& editing.

\section{Acknowledgment}

We thank the Werner Siemens Foundation (Werner Siemens-Stiftung) for its support of the Geothermal Energy and Geofluids (GEG.ethz.ch) Group at ETH Zurich, Switzerland. We also thank Dr. Anozie Ebigbo at Helmut Schmidt University for his generous time contribution and consultation regarding the reservoir impedance derivations and Qingrun Yang of the Ohio State University for useful discussions about the reservoir heat depletion solution.

ACT ELEGANCY, Project No 271498, has received funding from DETEC (CH), FZJ/PtJ (DE), RVO (NL), Gassnova (NO), BEIS (UK), Gassco AS and Statoil Petroleum AS, and is cofounded by the European Commission under the Horizon 2020 program, ACT Grant Agreement No 691712.

This work was also supported by HEATSTORE (170153-4401), which has been subsidized through the ERANET cofund GEOTHERMICA (Project n. 731117), from the European Commission, RVO (the Netherlands), DETEC (Switzerland), FZJ-PtJ (Germany), ADEME (France), EUDP (Denmark), Rannis (Iceland), VEA (Belgium), FRCT (Portugal), and MINECO (Spain).

Finally, we also thank the U.S. National Science Foundation Innovations at the Nexus of Food, Energy, and Water Systems (INFEWS) program (1739909), the U.S. National Science Foundation National Research Traineeship Program (1922666), and the Sloan Foundation.

\section{References}

Adams, B.M., Kuehn, T.H., Bielicki, J.M., Randolph, J.B., \& Saar, M.O. (2014). On the importance of the thermosiphon effect in $\mathrm{CPG}\left(\mathrm{CO}_{2}\right.$ plume geothermal) power systems. Energy, 69, 409-418. https://doi.org/10.1016/j.energy.2014.03.032

Adams, B.M., Kuehn, T.H., Bielicki, J.M., Randolph, J.B., \& Saar, M.O. (2015). A comparison of electric power output of $\mathrm{CO}_{2}$ Plume Geothermal (CPG) and brine geothermal systems for varying reservoir conditions. Applied Energy, 140, 365377. https://doi.org/10.1016/j.apenergy.2014.11.043

Adams, B.M., D. Vogler, T.H. Kuehn, J.M. Bielicki, N. Garapati, and M.O. Saar. (2020, in press). Heat Depletion in Sedimentary Basins and its Effect on the Design and Electric Power Output of CO2 Plume Geothermal (CPG) Systems, Renewable Energy, doi.org/10.1016/j.renene.2020.11.145.

Banks and Harris. (2018). Geothermal potential of foreland basins: A case study from the western Canadian sed basin. Geothermics

Bell, I. H., Wronski, J., Quoilin, S., \& Lemort, V. (2014). Pure and pseudo-pure fluid thermophysical property evaluation and the open-source thermophysical property library CoolProp. Industrial \& engineering chemistry research, 53(6), 24982508 .

Bistline, J. E., \& Blanford, G. J. (2020). Value of technology in the US electric power sector: Impacts of full portfolios and technological change on the costs of meeting decarbonization goals. Energy Economics, 86, 104694.

Carslaw, H.S., and Jaeger, J.C. (1986). "Ch. 13 - The Laplace transformation: Problems on the cylinder and sphere", in Conduction of Heat in Solids Second Edition (Oxford: Clarendon Press), pp 349-350.

Ezekiel, J., A. Ebigbo, B.M. Adams, M.O. Saar. (2020). Combining natural gas recovery and $\mathrm{CO}_{2}$-based geothermal energy extraction for electric power generation, Applied Energy, doi.org/10.1016/j.apenergy.2020.115012, 269, 115012.

Fleming, M.R., B.M. Adams, T.H. Kuehn, J.M. Bielicki, and M.O. Saar. (2020). Increased Power Generation due to Exothermic Water Exsolution in $\mathrm{CO} 2$ Plume Geothermal (CPG) Power Plants, Geothermics, doi.org/10.1016/j.geothermics.2020.101865 88, 101865.

Garapati, N., B.M. Adams, M.R. Fleming, T.H. Kuehn, and M.O. Saar. (2020). Combining Brine or CO2 Geothermal Preheating with Low-temperature Waste Heat: A Higher-efficiency Hybrid Geothermal Power System, Journal of CO2 Utilization, doi.org/10.1016/j.jcou.2020.101323, 42:101323.

IPCC (2018): Summary for Policymakers. In: Global Warming of $1.5^{\circ} \mathrm{C}$. An IPCC Special Report on the impacts of global warming of $1.5^{\circ} \mathrm{C}$ above pre-industrial levels and related global greenhouse gas emission pathways, in the context of strengthening the global response to the threat of climate change, sustainable development, and efforts to eradicate poverty [Masson-Delmotte, V., P. Zhai, H.-O. Pörtner, D. Roberts, J. Skea, P.R. Shukla, A. Pirani, W. Moufouma-Okia, C. Péan, R. Pidcock, S. Connors, J.B.R. Matthews, Y. Chen, X. Zhou, M.I. Gomis, E. Lonnoy, T. Maycock, M. Tignor, and T. Waterfield (eds.)]. World Meteorological Organization, Geneva, Switzerland, $32 \mathrm{pp}$.

Krey, V., Luderer, G., Clarke, L., \& Kriegler, E. (2014). Getting from here to there-energy technology transformation pathways in the EMF27 scenarios. Climatic change, 123(3), 369-382.

Kriegler, E., Weyant, J. P., Blanford, G. J., Krey, V., Clarke, L., Edmonds, J., ... \& van Vuuren, D. P. (2014). The role of technology for achieving climate policy objectives: overview of the EMF 27 study on global technology and climate policy strategies. Climatic Change, 123(3), 353-367.

Ramey, Jr., H.J. (1962). Wellbore Heat Transmission. Society of Petroleum Engineers.

Randolph, J.B. \& Saar, M.O. (2011). Combining geothermal energy capture with geologic carbon dioxide sequestration. Geophysical Research Letters, 38, L10401. https://dx.doi.org/10.1029/2011GL047265 
Randolph, J.B., Adams, B., Kuehn, T.H., \& Saar, M.O. (2012) Wellbore heat transfer in $\mathrm{CO}_{2}$-based geothermal systems. Geothermal Resources Council Transactions, 36: 549-554.

Ravilov, Marat (2019). Optimization of heat extraction within sedimentary reservoirs for $\mathrm{CO}_{2}$ Plume Geothermal (CPG) electricity generation. ETH Zurich. Master Thesis. https://doi.org/10.3929/ethz-b-000387209.

Sepulveda, N. A., Jenkins, J. D., de Sisternes, F. J., \& Lester, R. K. (2018). The role of firm low-carbon electricity resources in deep decarbonization of power generation. Joule, 2(11), 2403-2420.

U.S. Department of Energy Geothermal Technology Office. (2019). GeoVision: Harnessing the Heat Beneath Our Feet.

Stern, F. (2006). Mechanics of fluids and transport processes lecture notes. https://user.engineering.uiowa.edu/ fluids/Posting/ Lecture_Notes/Chapter6_Potential_Flow.pdf

Tester, J. W., Anderson, B. J., Batchelor, A. S., Blackwell, D. D., DiPippo, R., Drake, E. M., ... \& Veatch, R. W. (2006). The future of geothermal energy. Massachusetts Institute of Technology, 358.

Zhang, Y., Pan, L., Pruess, K., \& Finsterle, S. (2011). A time-convolution approach for modelling heat exchange between a wellbore and surrounding formatioon. Geothermics, 40, 261-266. 